\title{
Modified Implicit Method Embedded in a Two-Dimensional Space for Pricing Brazilian Interest Rate Derivatives
}

\author{
Allan Jonathan da Silva Jack Baczynski \\ Coordenação de Sistemas e Controle, CSC, LNCC, \\ 25651-075, Petrópolis, RJ \\ E-mail: allanjs@lncc.br, jack@lncc.br, \\ José V. M. Vicente \\ Banco Central do Brasil - Departamento de Pesquisa \\ 20071-000, Rio de Janeiro, RJ \\ E-mail: jose.valentim@ bcb.gov.br.
}

\begin{abstract}
$\underline{\text { ABSTRACT }}$
In the Brazilian fixed income market there is a standardized derivative product known as IDI (Interfinancial Deposits Index) Option, commonly used by corporations to manage risk. It is a financial Option of Asian type and, as such, the payoff depends of the path followed by this index. Among other works, [3] derived a closed form solution to price this contract based on a Martingale approach, assuming that the updating of the IDI is continuous. Based on the BM\&FBovespa definition we propose a discrete daily monitoring of the IDI, via a PDE approach and relaxing the continuity restriction.

We use the Vasicek [2] model, namely $d r=a(b-r) d t+\sigma d W$, to represent the random movements of the instantaneous short term interest rate. This Ornstein-Uhlenbeck stochastic process pull the short rate to a level $b$ at a rate $a$ together with a normally distributed random term $\sigma d W$, where $d W$ is the standard Brownian Motion. We assume that the contract value is a function of the time $t$, the short rate $r$ and the IDI $y$ only, and denote it by $V(r, y, t)$. So, we set up a portfolio containing one unit of the Option we want to price, and $\Delta$ units of another option of similar type and different maturity we use to hedge the position. Applying Ito's Lemma we have:

$d \Pi=\left(\frac{\partial V_{1}}{\partial t}+\frac{\sigma^{2}}{2} \frac{\partial^{2} V_{1}}{\partial r^{2}}-\Delta \frac{\partial V_{2}}{\partial t}-\Delta \frac{\sigma^{2}}{2} \frac{\partial^{2} V_{2}}{\partial r^{2}}\right) d t+\left(\frac{\partial V_{1}}{\partial y}-\Delta \frac{\partial V_{2}}{\partial y}\right) d y+\left(\frac{\partial V_{1}}{\partial r}-\Delta \frac{\partial V_{2}}{\partial r}\right) d r$
\end{abstract}

We assume that there is no risk premium. So, choosing $\Delta$ to eliminate the random term $d W$, reminding that the return on a risk-free portfolio must equal the risk-free rate to prevent financial arbitrage, and noting that $d y=0$ out of the monitoring points, we get the following PDE:

$$
\frac{\partial V}{\partial t}+\frac{1}{2} \sigma^{2} \frac{\partial^{2} V}{\partial r^{2}}+a(b-r) \frac{\partial V}{\partial r}=r V
$$

with final, boundary and jump conditions given by

$$
\begin{aligned}
V(r, y, T) & =\max (y-K, 0) \\
\frac{\partial V\left(r_{\text {min }}, y, t\right)}{\partial r} & =\frac{\partial V\left(r_{\text {max }}, y, t\right)}{\partial r}=0 \\
V\left(r, y_{i-1}, t_{i}^{-}\right) & =V\left(r, y_{i}, t_{i}^{+}\right), \text {where } \quad y_{i}=y_{i-1}\left(r_{i-1}+1\right) .
\end{aligned}
$$

The Finite Difference discretization of the PDE (1) is a well-known trouble in physics and in finance, specially when the sign of convective term changes and the volatility is very low, which are common 
facts in interest rate derivatives. In these cases the famous Crank-Nicolson scheme results in spurious oscillations, which makes financial engineers resort to the Up-wind scheme that stabilizes the solution at the cost of a lower order method and more expensive (unreal) contract values. We propose a full implicit discretization that guarantee a numerical solution with error of order $\mathcal{O}\left(\Delta r^{2}\right)$ and free of spurious oscillations, on the same lines as [1] for the stock-options Black-Scholes equation. We discretize the left hand side of (1) as usual via centered differences, and the right-hand side of (1) via $r(1-2 \alpha)\left(U_{j-1}^{n+1}+U_{j+1}^{n+1}\right)+r(4 \alpha-1) U_{j}^{n}$. The resulting tridiagonal matrix then shapes as

$$
A=\left[\frac{a(b-r)}{2 \Delta r}-\frac{0.5 \sigma^{2}}{\Delta r^{2}}+r-2 r \alpha ; \frac{1}{\Delta t}+\frac{\sigma^{2}}{\Delta r^{2}} ;-\frac{a(b-r)}{2 \Delta r}-\frac{0.5 \sigma^{2}}{\Delta r^{2}}+r-2 r \alpha\right] .
$$

Choosing $\alpha=0.5 r N^{\prime} \Delta r$, the scheme is stable if $\left.\Delta t<\left(2 r^{2} N^{\prime} \Delta r-r\right)\right)^{-1}$. Thus, A becomes diagonally dominant with real eigenvalues and an inverse with positive entries, in compliance with our stability and positivity-preserving requirements. The final condition (2) implies that a set of independent one-dimensional PDEs must be solved, one for each $y_{i}$ of the grid. The jump condition (4) performs the communication between the PDEs across the sample times, implemented by cubic spline interpolation.

To verify the method, we compare our full-implicit discretization in a bond pricing problem, with the closed form solution of [2] as a benchmark. As we are modeling interest rates, the region of interest is between, say, 0 and 0.4 . Some experiments strongly suggest, that in this region the prices solution independs of the value of the Dirichlet or Neumann boundary conditions. this allow us to choose homogeneous Neumann conditions for convenience. Fig. 1 and 2 shows that the solution is not affected by the boundary conditions and the difference tends to zero in the above range. By its turn, Fig. 3 shows

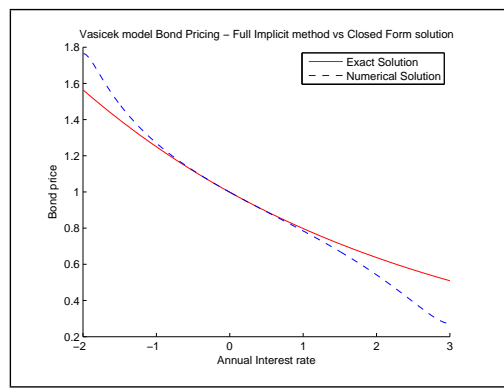

Figure 1: $a=0.1, b=0.5$, $\sigma=0.004, T=60$ days

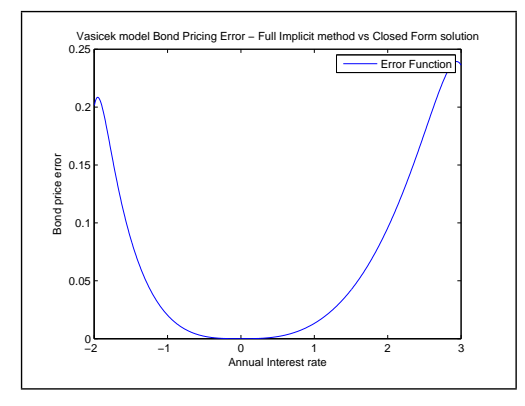

Figure 2: $a=0.1, b=0.5$, $\sigma=0.004, T=60$ days

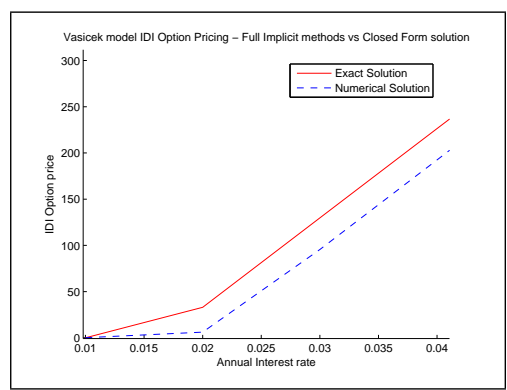

Figure 3: $a=0.1, b=0.5$, $\sigma=0.004, T=25$ days

that the IDI Option pricing methods based on continuous observations [3] lead to more expensive IDI Option prices, considering the Strike Price $K=100200$, the initial IDI equal to 100000 and the grid size $100 \times 200 \times 100$. It is known from basic financial mathematics that the discount bonds with daily or continuously compounded interest rates have similar values. Nonetheless this is not true when pricing standard lots of Asian option, which confirms the need of our results.

Keywords: Interest Rates Derivatives, PDE Option Pricing, Computational Finance

\section{References}

[1] M. Milev; A. Tagliani, Efficient implicit scheme with positivity preserving and smoothing properties, Journal of Computational and Applied Mathematics, 243 (2013) 1-9.

[2] O.A. Vasicek, "An Equilibrium Characterization of the Term Structure", Journal of Financial Economics, 5 (1977), 177-88.

[3] C.A. Vieira; P.L. Pereira, Closed Form Formula for the Price of the Options on the 1 Day Brazilian Interfinancial Deposits Index IDI1, em "Anais do XXII Congresso Brasileiro de Econometria" (Vol II), Campinas, 2000. 